\title{
Editorial
}

\section{Acknowledgement to Reviewers of Diagnostics in 2013}

Diagnostics Editorial Office, MDPI AG, Klybeckstrasse 64, CH-4057 Basel, Switzerland

Published: 28 February 2014

The editors of Diagnostics would like to express their sincere gratitude to the following reviewers for assessing manuscripts in 2013:

Bolondi, Luigi

Boon, Elles M.j.

Breydo, Leonid

Caudle, Michael

Chang, Wei-chiao

Chitty, Lyn

Choolani, Mahesh

Comhaire, Frank

Cosgrove, David

Dacher, Jean-nicolas

Dahdah, Nagib

Daneshmand, Siamak

Eftratiou, Androulla

Egi, Moritoki

Fernanda, Falcini

Fox, Susan H.

Ghoshal, Kalpana

Gilbert, Peter B.

Giubellino, Alessio

Graves, Edward

Hafeez, Shaista

Hagen, Gaute
Hanson, Kim

Hidalgo-Grass, Carlos

Iqbal, Samir

Jackson, Dr. Graham S.

Kambadakone, Avinash

Keenan, D. Barry

Kim, Joon-ho

Kim, Min Jung

Kim, Young-kee

Kwee, Thomas C.

Lee, Junghwa

Leidner, Bertil

Li, Qiang

Lu, Yujie

Mercuri, Nicola Biagio

Meredith, Ruby F.

Modlin, Irvin

Moeller, Soeren

Moise, Kenneth J.

Natarajan, Sathish

Nuutila, Pirjo

Ohuchida, Kenoki
Ombelet, Willem

Pertl, Barbara

Petitjean, Caroline

Pollock, Nira

Satoh, Asami

Schäfer, Helmut

Schneider, Jurgen

Sendra, Francisco

Stojanovic, Milan N.

Themelis, George

Tsuji, Yoshihisa

Vazquezbenitez, Gabriela.x.

Wang, Jiahui

Welling, Mick

White, Bruce A.

Wong, Tak-sing

$\mathrm{Wu}$, Jen-leih

$\mathrm{Wu}$, Jian

Wu, Jie

Yang, Yongfeng

Zhou, Qian

Zlotta, Alexandre R.

(C) 2014 by the authors; licensee MDPI, Basel, Switzerland. This article is an open access article distributed under the terms and conditions of the Creative Commons Attribution license (http://creativecommons.org/licenses/by/3.0/). 